20

\title{
Фотопроводящий ТГц детектор на основе новых функциональных слоев в многослойных гетероструктурах
}

\author{
() А.Э. Ячменев ${ }^{1,2}$, Д.В. Лаврухин ${ }^{1,2}$, Р.А. Хабибуллин ${ }^{1}$, Ю.Г. Гончаров ${ }^{2}$, И.Е. Спектор ${ }^{2}$, К.И. Зайцев ${ }^{2}$, \\ В.А. Соловьев ${ }^{3}$, С.В. Иванов ${ }^{3}$, Д.С. Пономарев ${ }^{1,2}$ \\ ${ }^{1}$ Институт сверхвысокочастотной полупроводниковой электроники им. В.Г. Мокерова РАН, \\ 117105 Москва, Россия \\ ${ }^{2}$ Институт общей физики им. А.М. Прохорова РАН, \\ 119991 Москва, Россия \\ ${ }^{3}$ ФТИ им. А.Ф. Иофффе, \\ 194021 Санкт-Петербург, Россия \\ e-mail: y.alex@runbox.com
}

Поступила в редакцию 31.12.2020 г.

В окончательной редакции 01.02.2021 г.

Принята к публикации 26.02.2021 г.

Проведено экспериментальное сравнение характеристик фотопроводящих антенн - детекторов терагерцевого (ТГц) излучения на основе сверхрешеточных гетероструктур InGaAs/InAs/InAlAs с различными типами упругих напряжений в слоях. На лабораторном импульсном спектрометре во временной области, используя образцы фотопроводящих детектирующих антенн с топологией „галстук-бабочка“, было выполнено сравнение ТГц сигналов, шумовых характеристик и отношений сигнал/шум при разной средней мощности оптического зондирования детекторов. Для фотопроводящих антенн (photoconductive antenna, PCA) на основе сверхрешеточных гетероструктур с упругими напряжениями как сжатия, так и растяжения в слоях во всем диапазоне мощностей лазерного излучения экспериментально продемонстрирована бо́льшая полоса детектирования по сравнению со случаем сверхрешеточных гетероструктур только напряжения сжатия. Таким образом, можно утверждать, что модификация свойств сверхрешеточных гетероструктур путем введения напряжений в кристаллическую решетку является универсальным и достаточно эффективным методом повышения характеристик РСА на их основе, что позволяет использовать эти РСА для построения практических систем ТГц спектроскопии и визуализации.

Ключевые слова: терагерцевая наука и техника, терагерцевая импульсная спектроскопия, спектрометр временной области, элементная база терагерцевой оптотехники, фотопроводящая антенна, фотопроводящая антенна-детектор, полупроводники, сверхрешеточная гетероструктура, молекулярно-пучковая эпитаксия, ПЭМ, неинвазивная медицина.

DOI: $10.21883 /$ OS.2021.06.50985.4-21

\section{Введение}

В последнее время активно развиваются новые подходы к решению прикладных задач медицинской диагностики и визуализации злокачественных новообразований различной нозологии и локализации, основанные на использовании терагерцового (ТГц) диапазона частот $[1,2]$. Среди уже полученных результатов можно выделить доказательства применимости ТГц подходов для ранней неинвазивной и интраоперационной диагностики злокачественных новообразований кожи и слизистой $[3,4]$, минимально-инвазивной и интраоперационной диагностики новообразований кишечника [5,6] и желудка [7], а также интраоперационной диагностики новообразований молочной железы [8] и головного мозга [9].

При использовании спектроскопии во временной области (time-domain spectrometer, TDS) генерация и детектирование ТГц излучения происходит когерентно, обеспечивая тем самым возможность одновременного получения амплитудной и фазовой информации об от- раженных или прошедших через объект исследований электромагнитных волнах. При этом TDS-системы при комнатной температуре характеризуются динамическим диапазоном по амплитуде вплоть до $55 \mathrm{~dB}$ (до $110 \mathrm{~dB}$ по мощности) при полосе частот от 0.1 до $4-5 \mathrm{THz}[10]$. Также следует отметить, что получение более полной информации о биологическом объекте при единичном измерении уже само по себе перспективно для решения задач медицинской диагностики.

Наиболее распространенными детекторами в импульсных ТГц системах являются фотопроводящие антенны (photoconductive antenna, PCA) [10-12] и электрооптические кристаллы [13-15]. Неоспоримым преимуществом первых является их универсальность и перестраиваемость за счет возможности согласования параметров фотопроводящего полупроводникового материала с длиной волны лазерной накачки, оптимизации топологии антенны и геометрии электродов, а также относительной простоты в изготовлении. Исторически наиболее широко используемым фотопроводящим мате- 
риалом стал выращенный при пониженной температуре GaAs $\left(E_{g}=1.42 \mathrm{eV}\right)[12]$, который работает с накачкой излучением с длиной волны 780-800 nm, излучаемой довольно сложной и громоздкой лазерной системой на основе кристалла Ti:Sapphire.

Очевидным недостатком GaAs с точки зрения применения компактных и недорогих волоконных лазеров ИК излучения является прозрачность данного материала для длинноволнового излучения. При этом волоконные лазеры находят все более широкое применение в ТГц оптоэлектронике (в системах спектроскопии с временным разрешением [16], создании стандартов частоты на частотных гребенках [17], измерении параметров излучения сторонних источников [18] и др.), а также становятся все надежнее и дешевле. Таким образом, разработка эффективных широкополосных детекторов ТГц излучения, совместимых с ИК излучением, востребована и актуальна для развития этого направления. Фотопроводник $\operatorname{In}_{x} \mathrm{Ga}_{1-x} \mathrm{As}$ имеет более узкую запрещенную зону по сравнению c GaAs, ширина которой может варьироваться от $0.92 \mathrm{eV}$ (для $x=0.38$ ) до $0.56 \mathrm{eV}$ (для $x=0.73)$ [19], что позволяет использовать оптическую накачку вплоть до $1.6 \mu \mathrm{m}[20,21]$. Однако применение его в чистом виде для РСА с приложением внешнего поля смещения к электродам затруднено из-за малого собственного сопротивления фотопроводника, что увеличивает вероятность теплового пробоя. Для преодоления этого ограничения применялась ионная имплантация [22,23], низкотемпературный рост с легированием атомами бериллия [24], однако одним из наиболее удобных и эффективных решений стало использование сверхрешеточных гетероструктур (superlattices heterostructures, SLS) InGaAs/InAlAs [25-28].

B настоящей работе мы предложили и исследовали гетероструктуры на основе сверхрешеток InGaAs/InAlAs, в функциональные слои которых были искусственно введены упругие (механические) напряжения путем изменения параметра кристаллической решетки за счет введения наноразмерных гетеровставок InAs в фотопроводящие слои InGaAs (SLS-1) и комбинации вставок InAs в InGaAs c одновременным изменением мольной доли индия в барьерах InAlAs (SLS-2). Ранее мы показали эффективность применения эпитаксиальных напряжений для управления динамикой фотовозбужденных носителей заряда, а также увеличения амплитуды генерации ТГц импульса в фотовольтаическом режиме работы $[27,29]$, в настоящей работе предложенный подход был расширен на создание РСА. Для этого на основе описанных структур были изготовлены образцы РСА, использовавшиеся для детектирования ТГц излучения (photoconductive antenna - detector, PCAD).

Экспериментально было показано, что введение упругих напряжений в сверхрешеточную гетероструктуру сильно влияет на характеристики детектора, в частности при работе с фемтосекундной лазерной накачкой на длине волны $780 \mathrm{~nm}$ позволяет на порядок повысить эффективность PCAD за счет одновременного уменьшения времени жизни носителей заряда, темнового тока и мощности шума детектора.

\section{Образцы и методы исследования}

В качестве материалов для изготовления РСА использовались SLS InGaAs/InAlAs с разным дизайном функциональных слоев. Всего методом молекулярнопучковой эпитаксии на подложках из полуизолирующего GaAs c ориентацией (001) были выращены две SLS. Для согласования параметров кристаллической решетки подложки GaAs и функциональных слоев использовался метаморфный буферный слой переменного состава InAlAs толщиной $\sim 0.7 \mu \mathrm{m}$.

Функциональные слои в SLS-1 состояли из 30 периодов $\operatorname{In}_{0.53} \mathrm{Ga}_{0.47} \mathrm{As} / \mathrm{InAs} / \mathrm{In}_{0.53} \mathrm{Ga}_{0.47} \mathrm{As} / \mathrm{In}_{0.52} \mathrm{Al}_{0.48} \mathrm{As}$, c центральной напряженной наноразмерной гетеровставкой InAs толщиной $3 \mathrm{~nm}$. Толщина каждого слоя $\mathrm{InGaAs}$ и InAlAs составляла 4.5 и $4.0 \mathrm{~nm}$ соответственно. Ранее мы показали возможность инженерии зонной структуры полупроводниковой гетероструктуры путем введения наноразмерных напряженных вставок InAs и GaAs [30] в квантовую яму InGaAs. В частности, было показано уменьшение эффективной массы носителей заряда при сохранении довольно высокой подвижности по сравнению с решеточно-согласованной структурой. Мы применили этот подход в настоящей работе, ожидая, что за время ТГц импульса большее число „легких“ фотовозбужденных носителей заряда успеет достигнуть электродов антенны. Помимо этого, гетероинтерфейсы между слоями с напряженной кристаллической решеткой также обладают повышенной шероховатостью по сравнению с гладкими гетероинтерфейсами у решеточносогласованных структур [31]. В многопериодной структуре большое количество таких гетероинтерфейсов увеличивает скорость рассеяния фотовозбужденных электронов на границах слоев, при этом подвижность в плоскости слоя остается высокой. За счет этого можно уменьшить времена релаксации носителей заряда (времена жизни) [27], темновой ток и мощность шума в PCAD без негативного влияния на полезный сигнал.

Гетероструктура SLS-2 имела аналогичную конструкцию функциональных слоев, за исключением того, что мольная доля индия в барьерных слоях $\operatorname{In}_{y} \mathrm{Al}_{1-y} \mathrm{As}$ была снижена до $y=38$. Наноразмерные гетеровставки InAs отличаются от $\operatorname{In}_{0.53} \mathrm{Ga}_{0.47} \mathrm{As}$ бо́льшим параметром кристаллической решетки, что приводит к появлению в фотопроводящих слоях со вставками упругих напряжений сжатия. По этой причине толщина слоев InAs была уменьшена до $1.5 \mathrm{~nm}$ - для компенсации упругих напряжений сжатия в фотопроводящем слое растягивающими напряжениями в барьерных слоях $\operatorname{In}_{0.38} \mathrm{Al}_{0.62} \mathrm{As}$ (с меньшим параметром решетки).

Исследование структурных особенностей выращенных образцов SLS проводилось методом просвечива- 
ющей электронной микроскопии (transmission electron microscopy, TEM) при ускоряющем напряжении $200 \mathrm{kV}$ на установке Titan Themis Z (производство FEI, CША) c корректором сферических аберраций конденсорной системы и 4 широкоугольных рентгеновских детекторов Super-X. Применялась стандартная методика приготовления поперечных срезов исследуемых гетероструктур при помощи резки и полировки образцов сфокусированным пучком Хе-плазмы с использованием двухлучевой системы Helios G4 PFIB UXe.

Для проведения экспериментов по детектированию ТГц излучения на основе выращенных SLS-1 и SLS-2 были изготовлены образцы PCAD: PCAD-1 и PCAD-2 соответственно. Рисунок металлизации был сформирован с помощью процесса фотолитографии с последующим термическим нанесением системы металлов $\mathrm{Ti} / \mathrm{Au}$ $(50 \mathrm{~nm} / 450 \mathrm{~nm})$ и далее „взрывом“ (lift-off) остаточной металлизации. Перед напылением электродов поверхность SLS была покрыта пассивирующим слоем $\mathrm{Si}_{3} \mathrm{~N}_{4}$ толщиной $230 \mathrm{~nm}$, в котором методом фотолитографии вскрывались „окна“ для обеспечения электрического контакта электродов антенны с поверхностью гетероструктуры. Использовалась топология „галстук-бабочка“ (bow-tie) с углом раструба $90^{\circ}$ и зазором между электродами $10 \mu \mathrm{m}$. Процесс изготовления образцов РСА подробно описан в работах [32,33].

Исследование образцов PCAD при разных значениях мощности оптического зондирования проводилось с помощью оригинального лабораторного импульсного ТГц спектрометра [32,34]. Источником излучения в спектрометре служила коммерческая PCA на основе низкотемпературного GaAs TERRA-8 (производство Menlo Systems, Германия), к которой прикладывалось переменное напряжение смещения с амплитудой $32 \mathrm{~V}$ и частотой повторения прямоугольных импульсов $20 \mathrm{kHz}$. Возникающий в исследуемом PCAD токовый сигнал усиливался малошумящим усилителем, а затем синхронно выпрямлялся. Источником ультракоротких оптических импульсов служил волоконный лазер с удвоением частоты выходного излучения EFOA-SH (производство АвестаПроект, Россия): центральная длина волны $780 \mathrm{~nm}$, частота повторения импульсов $f_{L}=65 \mathrm{MHz}$, длительность импульсов $\sim 100 \mathrm{fs}$. Мощность лазерного излучения в пучке накачки была фиксирована и составляла $10 \mathrm{~mW}$, в то время как в пучке зондирования регулируемый ослабитель позволял варьировать мощность в пределах $P_{\text {opt }}=0.3-12.0 \mathrm{~mW}$. Для согласования РСА-источника излучения и $\mathrm{PCAD}$ со свободным пространством использовались гиперполусферические кремниевые линзы диаметром $12 \mathrm{~mm}$ и высотой $7.1 \mathrm{~mm}$ (производство Batop, Германия); сбор и транспортировка ТГц излучения осуществлялись внеосевыми параболическими зеркалами диаметром $2^{\prime \prime}$ и фокусным расстоянием $4^{\prime \prime}$.

На каждую зарегистрированную волновую форму импульсного сигнала накладывалась оконная функция Тьюки (взвешенный косинус, tukey), выполнялось преобразование Фурье, в конце полученные спектры фазы

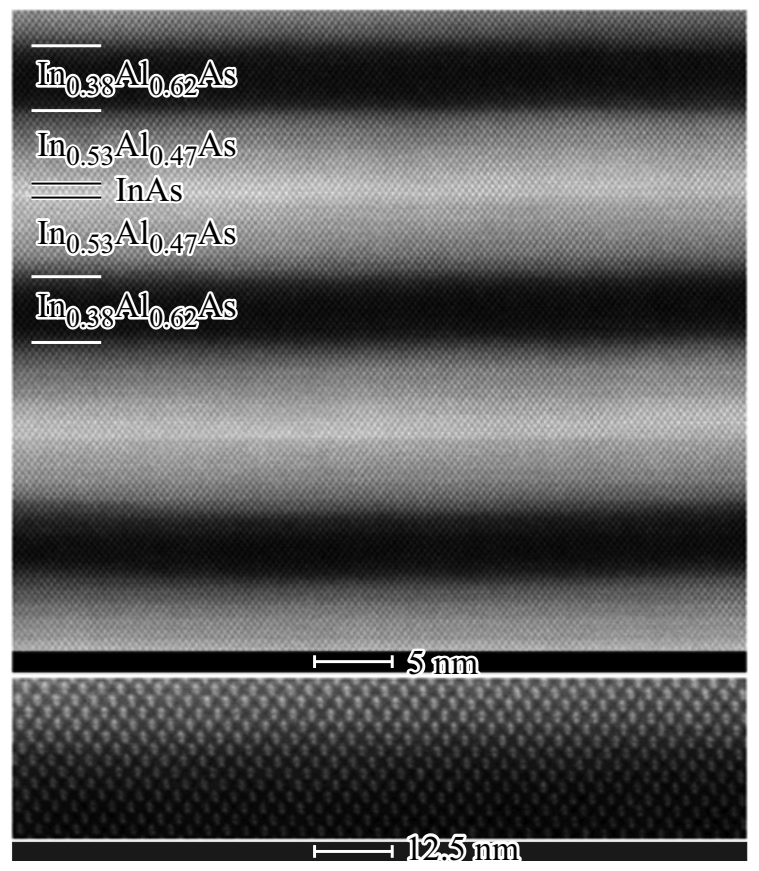

Рис. 1. Темнопольное изображение одного периода SLS2, сделанное с помощью ТЕМ. На вставке снизу показано увеличенное изображение гетероинтерфейса между слоями InGaAs и InAlAs.

и плотности мощности усредняются по 20 измерениям. Суммарное время регистрации спектра излучения с частотным разрешением $\sim 0.02 \mathrm{THz}$ для спектрометра составляло $\sim 20 \mathrm{~s}$, что было достигнуто, в том числе, использованием в линии оптической задержки быстродействующей $150 \mathrm{~mm}$ подвижки с линейным приводом (производство Daedal Parker, США) [36].

\section{Результаты исследований и их обсуждение}

Для исследования структуры с помощью ТЕМ была выбрана SLS-2 с более сложной конструкцией функциональных слоев, в которых присутствуют как упругие напряжения сжатия, так и растяжения. Изображение одного периода SLS-2 приведено на рис. 1. Видно, что толщины слоев соответствуют расчетным, а сами слои имеют четко выраженные гетерограницы. Это означает, что, несмотря на искусственно введенное рассогласование параметров решетки, выращенная гетероструктура обладает высокой степенью кристаллического совершенства. Отсутствие прорастающих дислокаций подтверждает тот факт, что упругие напряжения не релаксированы и кристаллическая решетка действительно является напряженной.

Зарегистрированные с помощью исследуемых PCAD спектры плотности мощности ТГц излучения приведены на рис. 2. Из-за различия в уровнях шумов детекторов 


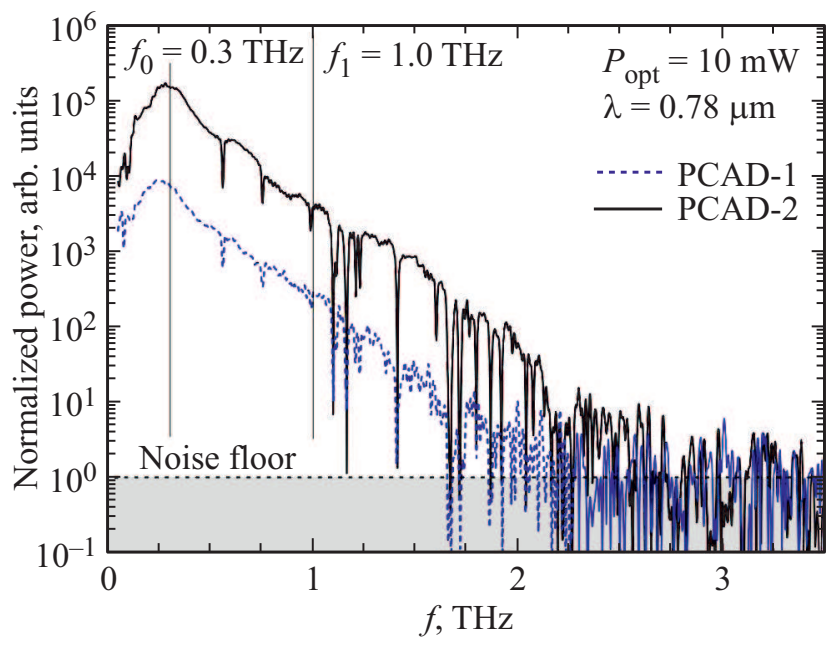

Рис. 2. Нормированные на шум спектры мощности ТГц излучения, зафиксированные антеннами-детекторами PCAD-1 (фиолетовая штриховая линия) и PCAD-2 (черная сплошная линия). Мощность зондирующего излучения $P_{\text {opt }}=10 \mathrm{~mW}$. Многочисленные узкие пики соответствуют резонансному поглощению излучения содержащимися в воздухе парами воды.

на основе SLS-1 и SLS-2 спектры для PCAD-1 и PCAD2 были отнормированы на среднеквадратичное значение мощности шума. Видно, что кривые для детекторов повторяют форму друг друга, отличаясь величиной спектр для PCAD-2 превосходит спектр для PCAD-1 в $\sim 20$ раз. Измеренные методом оптической накачки с зондированием (optical pump-probe) времена жизни носителей заряда для гетероструктур SLS-1 и SLS-2 составили $\tau_{c}=4.8 \mathrm{ps}$ и $2.5 \mathrm{ps}$ соответственно. Таким образом, разница в эффективности объясняется почти двукратным снижением времени жизни носителей заряда для PCAD2, обусловленным наличием в напряженных структурах SLS-2 дополнительных механизмов рассеяния на множестве гетероинтерфейсов между напряженными слоями.

Доминирующим источником шума в импульсных спектрометрах во временной области является шум Джонсона-Найквиста $[35,36]$, обратно пропорциональный среднему по времени сопротивлению детектоpa $\left\langle R_{a}\right\rangle$. Измеренные в отсутствие зондирующего излучения (темновые) электрические сопротивления детекторов и при малых значениях приложенного к зазору постоянного электрического поля $\left(\sim 10^{4} \mathrm{~V} / \mathrm{cm}\right)$ составили $R_{a 0}=4.7 \mathrm{k} \Omega$ и $7.8 \mathrm{k} \Omega$ для PCAD-1 и PCAD-2 соответственно. Шумовой сигнал детекторов, приведенный на рис. 3, регистрировался экспериментально путем блокировки детектора от пучка $\mathrm{THz}$ излучения металлической пластиной. Как видно из рисунка, среднеквадратическая мощность шума для обоих PCAD в пределах экспериментального разброса не зависит от мощности зондирующего излучения, а значит, форма кривых для отношения сигнал/шум (signal-to-noise ratio, SNR) определяется только зависимостью величины сигналов от мощности зондирующего излучения. Мы связываем зарегистрированную независимость шума от мощности зондирования с тем, что в PCAD с невысоким значением $R_{a 0}$ уменьшение электрического сопротивления, вызванное импульсом зондирования, уже не вносит заметного вклада в $\left\langle R_{a}\right\rangle$, т. е. $\left\langle R_{a}\right\rangle \simeq R_{a 0}$. Для подтверждения этой гипотезы был проведен контрольный эксперимент, в котором детекторы заменялись их пассивными электрическими эквивалентами - сопротивлениями $R_{a 0}$.

Известно, что в PCAD электрическое поле падающей $\mathrm{THz}$ волны приводит в движение свободные носители заряда, предварительно возбужденные в фотопроводящем слое зондирующим лазерным излучением. Мощность зондирующего излучения обычно невелика, поэтому с достаточно высокой точностью эффектами экранирования поля свободными зарядами можно пренебречь [32,33]. В таком случае величина фототока (регистрируемый спектрометром сигнал волновой формы) оказывается $\sim P_{\text {opt }}$, а зарегистрированная спектральная плотность мощности $\mathrm{THz}$ излучения $\sim P_{\text {орt }}^{2}$.

На рис. 4, $a$ в логарифмическом масштабе показано отношение сигнал/шум, вычисленное по экспериментальным данным для двух частот ТГц излучения при разной мощности зондирующего излучения. Видно, что для большей частоты детектируемого излучения разница в SNR между детекторами уменышается, что, вероятнее всего, связано эффектами интегрирования высокочастотных компонент регистрируемых в TDS волновых форм $\mathrm{THz}$ сигналов из-за того, что для обоих гетероструктур SLS-1 и SLS-2 времена жизни носителей заряда в фотопроводящих слоях InGaAs все же оказываются недостаточно малыми.

На рис. 4, $b$ (врезка) приведены значения отношения сигнал/шум для частоты $f_{0}=0.3 \mathrm{THz}$ в линейном масштабе с наложенными результатами полиномиальной аппроксимации. Видно, что кривые SNR отличаются

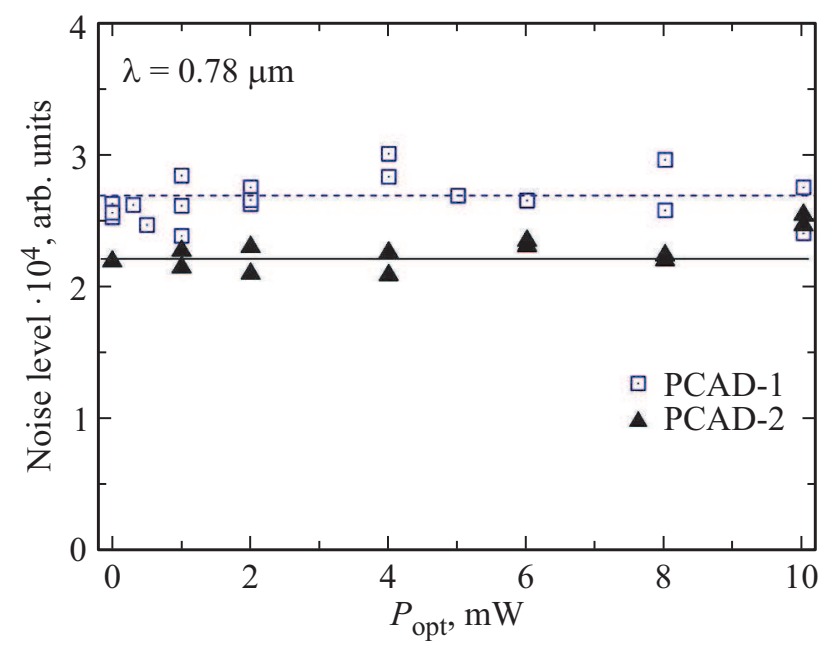

Рис. 3. Среднеквадратичные значения мощности шума для антенн-детекторов PCAD-1 (квадратные метки) и PCAD-2 (треугольные метки) при разной мощности зондирующего излучения $P_{\text {opt. }}$ Измерения проводились при перекрытом пучке ТГц излучения. 


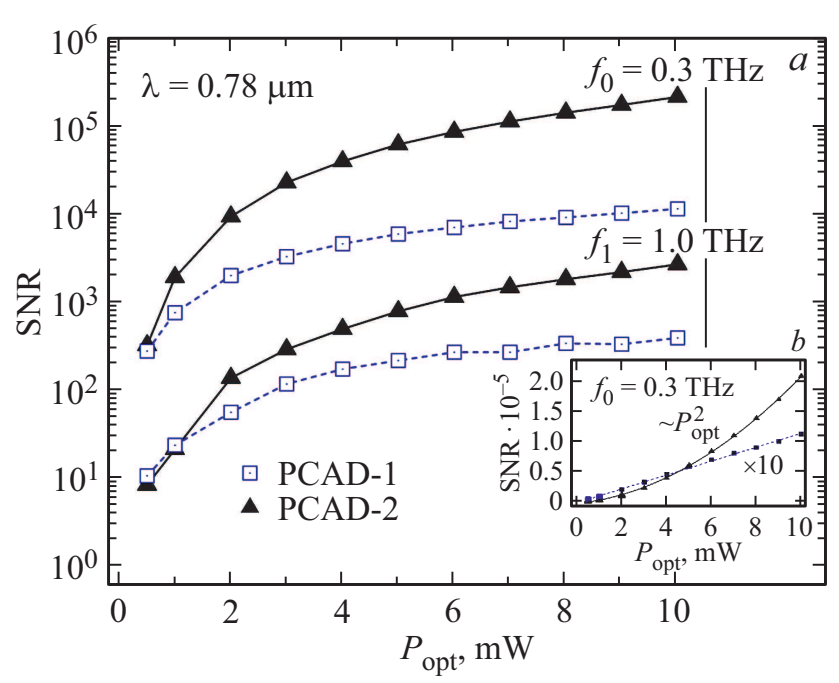

Рис. 4. Отношение сигнал/шум для PCAD-1 (квадратные метки) и PCAD-2 (треугольные метки): (a) в логарифмическом масштабе на частотах излучения $f_{0}=0.3 \mathrm{THz}$ и $f_{1}=1.0 \mathrm{THz}$; (b) в линейном масштабе на частоте излучения $f_{0}=0.3 \mathrm{THz}$ (масштаб для PCAD увеличен в 10 раз). В логарифмическом масштабе для наглядности метки соединены между собой отрезками. Образцы PCAD зондировались оптическим излучением с разной мощностью $P_{\text {opt }}$, мощность излучения накачки антенны-источника неизменна.

по характеру зависимости, при этом эффективность PCAD-2 выше, чем PCAD-1 во всем рассмотренном диапазоне $P_{\text {opt. }}$ В соответствии с вышеуказанными соображениями о работе PCAD кривая SNR для PCAD-2 хорошо описывается квадратичной зависимостью от $P_{\text {opt }}$ (показано сплошной линией). Отличие показателя степени для PCAD-1, скорее всего, связано с тем, что для областей малых сигналов (начальных участков кривых) обычно имеет место линейная связь значений функции с аргументом.

\section{Заключение}

В настоящей работе был экспериментально исследован подход к повышению эффективности фотопроводящих антенн-детекторов ТГц излучения (photoconductive antenna-detector, PCAD) на основе полупроводниковых сверхрешеточных гетероструктур (SLS) $\quad \operatorname{In}_{0.53} \mathrm{Ga}_{0.47} \mathrm{As} / \mathrm{In}_{0.52} \mathrm{Al}_{0.48} \mathrm{As}, \quad$ связанный $\mathrm{c}$ введением в SLS упругих напряжений за счет изменения параметров решетки слоев SLS. Выращенные SLS отличались методом создания упругих напряжений. Первая гетероструктура включала наноразмерные вставки InAs в InGaAs, создающие в кристаллической решетке напряжения сжатия. Вторая структура помимо наноразмерных вставок InAs в InGaAs содержала уменьшенную мольную долю In в барьерных слоях InAlAs, что приводило к одновременному формированию внутренних напряжений растяжения. На основе SLS-1 и SLS-2 мы изготовили образцы детекторов PCAD-1 и PCAD-2.
Для образцов детекторов PCAD-1 и PCAD-2 проводились измерения спектров плотности мощности ТГц излучения, а также зависимости уровня шума и отношения сигнал/шум (signal-to-noise ratio, SNR) от оптической мощности зондирующего излучения $P_{\text {opt. }}$ Сравнительный анализ показал, что во всем диапазоне $P_{\text {opt }}$ от 0.5 до $10.0 \mathrm{~mW}$ SNR для PCAD-2 более чем на порядок превосходит значение для PCAD-1, что мы связываем с более эффективной конструкцией функциональных слоев сверхрешетки, обеспечивающей бо́льшую скорость рассеяния фотовозбужденных электронов за счет большего числа гетероинтерфейсов между упруго напряженными решетками без существенного уменьшения подвижности в плоскости фотопроводящих слоев.

Таким образом, предложенный нами подход к улучшению характеристик фотопроводящих материалов с помощью введения упругих напряжений в кристаллическую решетку многослойных гетероструктур является достаточно эффективным и универсальным за счет возможности применения в конструкции любой многослойной гетероструктуры. Причем наибольший эффект может быть достигнут использованием комбинации слоев с компенсирующими друг друга разнонаправленными напряжениями, что позволяет увеличить количество формируемых гетеропереходов между напряженными слоями без накапливания напряжений в одном направлении и последующей их релаксации с образованием дислокаций.

\section{Благодарности}

Авторы выражают благодарность профессорам А. Абакумову и Я. Шаховой за измерения ПЭМ, которые проводились на базе ЦКП „Визуализация высокого разрешения“ Сколковского института науки и технологий.

\section{Финансирование работы}

Работа была выполнена при финансовой поддержке гранта РНФ 18-79-10195.

\section{Конфликт интересов}

Авторы заявляют, что у них нет конфликта интересов.

\section{Список литературы}

[1] Zaytsev K.I., Dolganova I.N., Chernomyrdin N.V., Katyba G.M., Gavdush A.A., Cherkasova O.P., Komandin G., Shchedrina M.A., Khodan A.N., Ponomarev D.S., Reshetov I.V., Karasik V., Skorobogatiy M., Kurlov V.N., Tuchin V.V. // J. Optics. 2019. V. 22. N 1. P. 013001.

[2] Smolyanskaya O.A., Chernomyrdin N.V., Konovko A.A., Zaytsev K.I., Ozheredov I.A., Cherkasova O.P., Nazarov M.M., Guilleti J.P., Kozlova S.A., Kistenev Yu.V., Coutaz J.-L., Mounaix P., Vaks V.L., Son J.-H., Cheon H., Wallace V.P., Feldman Yu., Popov N.I., Tuchin V.V. // Progress in Quant. Electron. 2018. V. 62. P. 1. 
[3] Woodward R.M., Wallace V.P., Pye R.J., Cole B.E., Arnone D.D., Linfield E.H., Pepper M. // J. Investigative Dermatology. 2003. V. 120. N 1. P. 72.

[4] Wallace V.P., Fitzgerald A.J., Shankar S., Flanagan N., Pye R., Cluff J., Arnone D.D. // British J. Dermatology. 2004. V. 151. N 2. P. 424

[5] Reid C.B., Fitzgerald A., Reese G., Goldin R., Tekkis P., O'Kelly P.S., Pickwell- MacPherson E., Gibson A.P., Wallace V.P. // Physics in Medicine \& Biology. 2011. V. 56. N 14. P. 4333.

[6] Doradla P., Alavi K., Joseph C.S., Giles R.H. // J. Biomedical Optics. 2013. V. 18. N 9. P. 090504.

[7] Hou D., Li X., Cai J., Ma Y., Kang X., Huang P., Zhang G. // Physics Medicine \& Biology. 2014. V. 59. N 18. P. 5423.

[8] Fitzgerald A.J., Wallace V.P., Jimenez-Linan M., Bobrow L., Pye R.J., Purushotham A.D., Arnone D.D. // Radiology. 2006. V. 239. N 2. P. 533.

[9] Oh S., Kim S.H., Ji Y.B., Jeong K., Park Y., Yang J., Park D.W., Noh S.K., Kang S.G., Huh Y.M., Son J.H., Suh J.S. // Biomedical Optics Express. 2014. V. 5. N 8. P. 2837.

[10] Yachmenev A.E., Lavrukhin D.V., Glinskiy I.A., Zenchenko N.V., Goncharov Y.G., Spektor I.E., Khabibullin R.A., Otsuji T., Ponomarev D.S. // Opt. Eng. 2019. V. 59. N 6. P. 061608

[11] Lu P.K., Turan D., Jarrahi M. // Optics Express. 2020. V. 28. P. 26324-26335.

[12] Yachmenev A.E., Pushkarev S.S., Reznik R.R., Khabibullin R.A., Ponomarev D.S. // Progress in Crystal Growth and Characterization of Materials. 2020. V. 66. N 2. P. 100485.

[13] Nagai M., Tanaka K. // Appl. Phys. Lett. 2004. V. 85. P. 3974.

[14] Ilyakov I.E., Kitaeva G.Kh., Shishkin B.V., Akhmedzhanov R.A. // Opt. Lett. 2017. V. 42. P. 1704-1707.

[15] Leitenstorfer A., Hunsche S., Shah J., Nuss M.C., Knox W.H. // Appl. Phys. Lett. 1999. V. 74. P. 1516.

[16] Zhang Y., Zhang X., Li S., Gu J., Li Y., Tian Z., Ouyang C., He M., Han J., Zhang W. // Sci. Rep. 2016. V. 6. P. 1-6.

[17] Nagatsuma T., Horiguchi S., Minamikata Y., Yoshimizu Y., Hisatake S., Kuwano S., Yoshimoto N., Terada J., Takahashi H. // Optics Express. 2013. V. 21. P. 23736-23747.

[18] Barbieri S., Ravaro M., Gellie P., Santarelli G., Manquest C., Sirtori C., Khanna S.P., Linfield E.H., Davies A.G. // Nature Photonics. 2011. V. 5. P. 306-313.

[19] Ilyakov I.E., Shishkin B.V., Malevich V.L., Ponomarev D.S., Galiev R.R., Yachmenev A.E., Akhmedzhanov R.A., Khabibullin R.A. // Appl. Phys. Lett. (under review).

[20] Takazato A., Kamakura M., Matsui T., Kitagawa J., Kadoya Y. // Appl. Phys. Lett. 2007. V. 91. P. 011102.

[21] Huber R., Brodschelm A., Tauser F., Leitenstorfer A. // Appl. Phys. Lett. 2000. V. 76. N 22. P. 3191.

[22] Chimot N., Mangeney J., Mounaix P., Tondusson M., Blary K., Lampin J.F. // Appl. Phys. Lett. 2006. V. 89. P. 083519.

[23] Suzuki M., Tonouchi M. // Appl. Phys. Lett. 2005. V. 86. P. 163504.

[24] Han S.-P., Ko H., Kim N., Ryu H.-C., Lee C.W., Leem Y.A., Lee D., Jeon M.Y., Noh S.K., Chun H.S., Park K.H. // Opt. Lett. 2011. V. 36. N 16. P. 3094.

[25] Ospald F., Maryenko D., Klitzing K., Driscoll D.C., Hanson M.P., Lu H., Gossard A.C., Smet J.H. // Appl. Phys. Lett. 2008. V. 92. P. 131117.

[26] Dietz R.J., Globisch B., Roehle H., Stanze D., Göbel T., Schell M. // Opt. Express. 2014. V. 22. P. 19411.
[27] Ponomarev D.S., Gorodetsky A., Yachmenev A.E., Pushkarev S.S., Khabibullin R.A., Grekhov M.M., Zaytsev K.I., Khusyainov D.I., Buryakov A.M., Mishina E.D. // J. Appl. Phys. 2019. V. 125. N 15. P. 151605.

[28] Ponomarev D.S., Yachmenev A.E., Glinskiy I.A., Khabibullin R.A., Khusyainov D.I., Buryakov A.M., Mishina E.D. // Proc. SPIE. 2019. V. 11457. P. 114571A.

[29] Ospald F., Maryenko D., Klitzing K., Driscoll D.C., Hanson M.P., Lu H., Gossard A.C., Smet J.H. // Appl. Phys. Lett. 2008. V. 92. P. 131117.

[30] Ponomarev D.S., Yachmenev A.E., Glinskiy I.A., Khabibullin R.A., Khusyainov D.I., Buryakov A.M., Mishina E.D. /I Proc. SPIE. 2019. V. 11457. P. 114571A.

[31] Buryakov A., Khusyainov D., Mishina E., Yachmenev A., Khabibullin R., Ponomarev D. // MRS Advances. 2019. V. 4. N 1. P. 15-20.

[32] Kulbachinskii V.A., Yuzeeva N.A., Galiev G.B., Klimov E.A., Vasil'evskii I.S., Khabibullin R.A., Ponomarev D.S. // Semicond. Sci. Technol. 2012. V. 27. P. 035021.

[33] Chen Y.C., Bhattacharya P.K., Singh J. // J. Crystal Growth. 1991. V. 111. P. 228-232.

[34] Lavrukhin D.V., Yachmenev A.E., Glinskiy I.A., Khabibullin R.A., Goncharov Y.., Ryzhii M., Otsuji T., Spector I.E., Shur M., Skorobogatiy M., Zaytsev K.I., Ponomarev D.S. // AIP Advances. 2019. V. 9. P. 015112.

[35] Lavrukhin D.V., Yachmenev A.E., Pavlov A.Yu., Khabibullin R.A., Goncharov Yu.G., Spektor I.E., Komandin G.A., Yur-chenko S.O., Chernomyrdin N.V., Zaytsev K.I., Ponomarev D.S. // Semicond. Sci. Technol. 2019. V. 34. P. 034005.

[36] Лаврухин Д.В., Галиев Р.Р., Павлов А.Ю., Ячменев А.Э., Майтама М.В., Глинский И.А., Хабибуллин Р.А., Гончаров Ю.Г., Зайцев К.И., Пономарев Д.С. // Опт. и спектр. 2019. T. 126. B. 5. C. 663 .

[37] Zhang X.C., Jepsen P.U., Jacobsen R.H., Keiding S.R. // J. Opt. Soc. Am. B. 1996. V. 13. N 11. P. 2424.

[38] Wang N., Jarrahi M. // J. Infrared Millim. Terahertz Waves. 2013. V. 34. N 9. P. 519-528. 